\title{
Efficient Estimation of Dynamic Density Functions with Applications in Data Streams
}

\author{
Abdulhakim Qahtan ${ }^{1}$, Suojin Wang ${ }^{2}$, and Xiangliang Zhang ${ }^{3 凶}$ \\ 1 Qatar Computing Research Institute (QCRI), HBKU, Qatar \\ aqahtan@hbku.edu.qa \\ 2 Department of Statistics, TAMU, Texas 77843-3143, USA \\ sjwang@stat. tamu.edu \\ 3 CEMSE, King Abdullah University of Science and Technology (KAUST), KSA \\ xiangliang.zhang@kaust.edu.sa
}

\begin{abstract}
Recently, many applications such as network monitoring, traffic management and environmental studies generate huge amount of data that cannot fit in the computer memory. Data of such applications arrive continuously in the form of streams. The main challenges for mining data streams are the high speed and the large volume of the arriving data. A typical solution to tackle the problems of mining data streams is to learn a model that fits in the computer memory. However, the underlying distributions of the streaming data change over time in unpredicted scenarios. In this sense, the learned models should be updated continuously and rely more on the most recent data in the streams. In this chapter, we present an online density estimator that builds a model called KDE-Track for characterizing the dynamic density of the data streams. KDE-Track summarizes the distribution of a data stream by estimating the Probability Density Function (PDF) of the stream at a set of resampling points. KDE-Track is shown to be more accurate (as reflected by smaller error values) and more computationally efficient (as reflected by shorter running time) when compared with existing density estimation techniques. We demonstrate the usefulness of KDE-Track in visualizing the dynamic density of data streams and change detection. Keywords: Dynamic Density Estimation, Kernel Density Estimation, Data Streams; Adaptive Resampling; Linear Interpolation
\end{abstract}

\section{Introduction}

Recent advances in computing technology allow for collecting vast amount of data that arrive continuously in data streams. Examples of data streams can be found in fields such as sensor networks, mobile data collection platform, and network traffic. The data need to be processed and analyzed once they arrive. However, the unbounded, rapid and continuous arrival of data streams disallow the usage of traditional data mining techniques. Therefore, the development of online algorithms for processing data streams becomes highly important.

Density estimation has been widely used in various applications. Estimating the Probability Density Function (PDF) for a given data set provides knowledge 
about the underlying distribution of the data. Consequently, dense regions can be recognized as clusters and quantities such as medians and centers of clusters can be computed [1]. By contrast, sparse regions are reported as outliers that can be used for fault detection, e.g., in sensor networks [2. Moreover, the estimated dynamic density can be visualized to help on placing taxicabs in places with high pickup rate at certain period of time [3], reducing ambulance emergencies response time [4] and reflecting people's interest at a particular location for specific seasons [5].

Estimating the dynamic density that comes with evolving streams needs to address the following challenges. First, the data distribution changes dynamically in an unpredictable fashion. Second, an anytime-available model should be efficiently updated to allow real-time monitoring of the density. Third, the spatial non-uniformity of data distribution requires higher/lower resolutions in dense/sparse areas, respectively, so that the estimation is accurate to catch the details.

Most of the existing approaches for estimating the density of data streams are based on the Kernel Density Estimation (KDE) method due to its advantages for estimating the true density [6]. Given a set of samples, $S=\left\{\boldsymbol{x}_{1}, \boldsymbol{x}_{2}, \ldots, \boldsymbol{x}_{n}\right\}$ with $\boldsymbol{x}_{j} \in R^{d}$, KDE estimates the density at a point $\boldsymbol{x}$ as:

$$
\hat{f}(\boldsymbol{x})=\frac{1}{n} \sum_{j=1}^{n} K_{h}\left(\boldsymbol{x}, \boldsymbol{x}_{j}\right),
$$

where $K_{h}\left(\boldsymbol{x}, \boldsymbol{x}_{j}\right)$ is a kernel function, which is usually a radially symmetric unimodal function that integrates to 1. Eq. (1) shows that KDE uses all the data samples to estimate the PDF at any given point. For online density estimation of data stream, i.e., estimating the density of every arriving data sample, KDE has quadratic time complexity with respect to (w.r.t.) the stream size. Also, the space requirement for KDE significantly increases with the dataset size.

In Section 3 of this chapter, we introduce our model that is called KDETrack, to model the data distribution as a set of resampling points with their estimated PDF. To guarantee the estimation accuracy and to lighten the load on the model, an adaptive resampling strategy is employed to control the number of resampling points, i.e., more points are resampled in the areas where the PDF has a larger curvature, while less number of points are resampled in the areas where the function is approximately linear. In order to overcome the quadratic time complexity of KDE when evaluating the PDF for each new observation, linear interpolation is used with $\mathrm{KDE}$ for online density estimation. It therefore has advantages of evaluating the PDF for any new observation in linear time complexity and space complexity w.r.t. the number of resampling points. Evaluating the PDF for all received observations will then take linear time w.r.t. the stream size compared with the quadratic time complexity of KDE. To timely track the evolving density, we use a sliding window strategy in KDE-Track to estimate the density using the most recent data samples.

The KDE-Track has unique properties as follows:

(1) it generates density functions that are available to visualize the dynamic 
density of data streams at any time. After receiving one streaming data sample $\boldsymbol{x}_{t}$, KDE-Track updates the PDF of the data stream and also estimates $f\left(\boldsymbol{x}_{t}\right)$;

(2) it has linear time and space complexities w.r.t. the model size for maintaining the dynamic PDF of data stream upon the arrival of every new sample. It is thus $8-85$ times faster than traditional KDE depending on the window size; (3) the estimation accuracy is achieved by adaptive resampling and optimized bandwidth $(h)$, which also address the spatial non-uniformity issue of data streams.

In Section 4 of this chapter, we evaluate the most popular density estimators, as well as KDE-Track in different scenarios of data streams. Advantages and disadvantages of these evaluated methods are discussed based on the comparison of their performance. The obtained dynamic density can be applied to diverse problems. Section 5 of this chapter firstly presents a density visualization example, which displays the real-time dynamic traffic distribution in the New York Taxi streams where interesting patterns of community behavior are discovered. The second application is for unsupervised change detection, where changes are usually detected by comparing the distribution in a current (test) window sliding over the data stream with a fixed reference window that contains data arrived after the last detected change. The third application is outlier detection in data streams, which is a straightforward application of estimated density. Data samples, which have small PDF values compared to other points, are more likely to be outliers. Compared with other outlier detection methods, density-based approach is shown to report less false positives. This application is omitted in this chapter due to space limitation. Interested readers are refereed to [7].

\section{Related Work}

\subsection{Dynamic Density}

The design of dynamic density estimator should not only take into consideration of the constraints of using limited memory and processing the data in real time (899) due to the nature of streams, but also the dynamic changes of the underlying density function over time. To reduce the computational cost and space requirement of $\mathrm{KDE}$, methods have been proposed based on kernel merging, sampling or space partitioning. Kernel merging is used in [110] and 11] where a specific number of kernels are maintained through merging two or more kernels. Each kernel summarizes a cluster of similar samples. A new arriving sample can either fall into one existing kernel or trigger a new kernel. Two kernels are merged if the number of kernels exceeds the specified number. Methods that utilize this concept include M-Kernel [1, Cluster Kernels (CK) [10] and AKDE [11. Another kernel method emerges based on clustering by Self-Organizing Maps (SOM) 12. Only trained SOM neurons are utilized in density estimation, rather than the whole set of kernels. In order to train the neurons and to minimize the time complexity, a data stream is considered as a sequence of disjoint windows where data in each window are assumed to have the same distribution. 
Table 1: Summary of the key characteristics of the density estimators (MV = Multivariate data, $\mathrm{RS}=$ Random Sampling, GS = Group Selection, $\mathrm{SS}=$ Sort Selection, $\mathrm{RI}=$ Rotation Invariant and BU = Batch Update).

\begin{tabular}{|l|c|c|c|c|c|c|c|}
\hline Method & MV & $\begin{array}{c}\text { Data } \\
\text { streams }\end{array}$ & $\begin{array}{c}\text { Bandwidth } \\
\text { selection }\end{array}$ & $\begin{array}{c}\text { Bandwidth of } \\
\text { each dim in MV }\end{array}$ & $\begin{array}{c}\text { Data points } \\
\text { reduction }\end{array}$ & $\begin{array}{c}\text { MV Kernel } \\
\text { function }\end{array}$ & $\begin{array}{c}\text { Online } \\
\text { update }\end{array}$ \\
\hline CK 10 & No & Yes & Normal rule & N/A & Merge kernels & N/A & Yes \\
M-Kernels 1] & No & Yes & Normal rule & N/A & Merge kernels & N/A & Yes \\
SOMKE [12 & Yes & Yes & Normal rule & Different & Trained neurons & MV & BU \\
FFT-KDE 19 & Yes & Yes & Normal rule & Different & none & MV & No \\
KDE[20] & Yes & Yes & Normal rule & Different & none & Product & No \\
kd-tree [15 & Yes & No & Cross validation & Fixed & none & RI & No \\
RS, GS, SS 13 & Yes & No & User input & Fixed & sampling & RI & No \\
MPLKernels [14] & Yes & Yes & Normal rule & Different & sampling & Product & Yes \\
KDE-Track 18 & Yes & Yes & plug in & Different & none & Product & Yes \\
\hline
\end{tabular}

Sampling was used in [13 to reduce the number of kernels while guaranteeing an $\epsilon$-approximation of the density function. The authors studied random sampling and proposed group-selection and sort-selection, which achieve the same accuracy as random sampling but with a smaller number of samples.

Space partitioning is also used to reduce the computational cost of KDE. A $k d$-tree structure is used in [14] and [15] where the leaves contain a small number of kernels and each internal node contains a statistical summary about the subspace represented by that node. Estimating the density at any given point involves depth-first traversal of the tree where only close-by nodes will be visited. Grid-based methods were presented in 1617 for static datasets. They concentrate on the best setting of the bin width using a fixed number of resampling points. This approach will not work for data streams as the data are not available in advance and the range of the data is changing over time.

KDE-Track [18 differs from the above-mentioned methods by updating the estimated density with the contribution of each new arriving sample. Hence, it provides any time available density values, which can be used for visualizing the density. The model size is controlled by adaptive resampling, rather than reducing the number of used kernels in [1|11|10|12]13|14. The estimation error is thus minimized. In addition, it is deployed with an accurate bandwidth selection method, which improves the density estimation significantly.

\subsection{Change Detection}

Change detection is relevant to a wide range of applications such as intrusion detection in computer networking [21, suspicious motion detection in vision systems [22], studying the effects of nuclear radiation on the environment [23], and online clustering and classification. For example, change detection can be used in online classification for reporting when the classifier should be re-trained (only if a change in the data stream is observed). The problem of change detection has been widely studied and referred to as data evolution [24, event detection 25] or change-point detection [2623].

Unsupervised window-based change detection is based on comparing the distribution in a current stream window with a reference distribution [27/28/23/29/30], where density estimation techniques and divergence metrics are essential to 
Table 2: Summary of change detection methods (MV = Multivariate).

\begin{tabular}{|c|c|c|c|c|}
\hline Technique & MV & Data streams & Compare Distribution/Prediction & Threshold Settings \\
\hline kdq-tree [28] & \begin{tabular}{|l|} 
Yes \\
\end{tabular} & Yes & Compare Distribution & Bootstrap \\
\hline PCA-SPLL 29 & Yes & No & Compare Distribution & Fixed \\
\hline KLIEP 26] & Yes & Yes & Compare Distribution & Fixed \\
\hline Kifer 23. & No & Yes & Compare Distribution & Bootstrap \\
\hline ADWIN 27 & No & Yes & other & Dynamic \\
\hline Density test 30 & Yes & No & Compare Distribution & Bootstrap \\
\hline $\mathrm{CF} 32$ & No & Yes & Prediction & Fixed \\
\hline CD-Area 33 & Yes & Yes & Compare Distribution & Dynamic \\
\hline
\end{tabular}

model and compare the distributions. Dasu et al. 28 used the kdq-tree data structure to model data distribution and presented a 3 -step process for change detection: 1) updating the test distribution over the current window; 2) computing the change-score between the test and reference distributions; and 3) emitting an alarm signal if the change-score reaches the threshold specified using bootstrap sampling and the permutation test used in [23]. Kawahara and Sugiyama [26] used the density-ratio estimation that is based on the KullbackLeibler Importance Estimation Procedure (KLIEP) to model data distribution. The method's complexity is quadratic w.r.t. the window size. An online version of KLIEP was studied in 26] and 31.

A statistical test, called the density test [30], determines whether the newly observed data $S^{\prime}$ are sampled from the same underlying distribution as the reference dataset $S$. The change detection summarized above has the limitation of user-based settings of key parameters, which requires users knowledge or they have high computational that makes them unusable for online change detection in data streams.

Table 2 summarizes the key characteristics of the existing methods for change detection. In Section 5, a PCA-based change detection framework will be presented 33 . The framework compares densities estimated by KDE-Track on selected principal components and dynamically adjusts the threshold for reporting changes, such that the false alarms are reduced and detection rate is improved.

\section{KDE-Track: Dynamic Density Estimation}

\subsection{Theoretical Bases of Density Estimation}

We first discuss the traditional KDE and its related issues, e.g., selection of kernel functions and smoothing parameter (bandwidth), and complexity.

KDE estimates the density $\hat{f}(\boldsymbol{x})$ by Eq. (1). For the case of univariate data, Eq. (1) is written as:

$$
\hat{f}(x)=\left(\frac{1}{n h}\right) \sum_{j=1}^{n} K\left(\frac{x-x_{j}}{h}\right) .
$$

For the two dimensional samples, where $\boldsymbol{x}_{j}=\left(x_{1 j}, x_{2 j}\right)^{T} \in R^{2}$, kernel functions $K_{h}\left(\boldsymbol{x}, \boldsymbol{x}_{j}\right)$ are defined as $\frac{1}{h_{1} h_{2}} K\left(\frac{x_{1}-x_{1 j}}{h_{1}}, \frac{x_{2}-x_{2 j}}{h_{2}}\right)$, where $h_{i}$ is the smoothing parameter, called the bandwidth, on dimension $i$ [6]. 
A popular kernel function in case of multivariate data is called the multiplicative (product) kernel [6], which uses the product of univariate kernel functions on each dimension, and in the 2 dimensional case computes $\hat{f}(\boldsymbol{x})$ as:

$$
\hat{f}(\boldsymbol{x})=\frac{1}{n} \sum_{j=1}^{n} \prod_{i=1}^{2}\left\{\frac{1}{h_{i}} K\left(\frac{x_{i}-x_{j i}}{h_{i}}\right)\right\} .
$$

Another option is to use the orientation-invariant kernel function [13] and [15], which is:

$$
\hat{f}(\boldsymbol{x})=\left(\frac{1}{n h^{2}}\right) \sum_{j=1}^{n} K\left(\frac{\left\|\boldsymbol{x}-\boldsymbol{x}_{j}\right\|}{h}\right) .
$$

This kernel function assumes that the data variation along all the dimensions is the same, which may fail to capture densities of arbitrary shapes.

KDE related issues. The choice of a kernel function is relatively unimportant provided that a kernel function is continuous with finite support 20]. It is recommended that the selected kernel is smooth, clearly unimodal and symmetric about the origin 6. In the study of this chapter, we choose the multiplicative Epanechnikov kernel where the same univariate kernel function $K(x)=\frac{3}{4}\left(1-x^{2}\right) I_{[-1,1]}(x)$ is used in each dimension with a different bandwidth value. We use the Epanechnikov kernel because of its asymptotically-optimal efficiency among all other kernel functions 34 .

The estimation accuracy of KDE is mainly affected by the bandwidth value [6]20. A large bandwidth value over-smooths the density function curve and hides a lot of useful information, while a small bandwidth value makes the density function's curve too fluctuated. A general rule for selecting the bandwidth is to decrease the bandwidth value $(h \rightarrow 0)$ as the number of samples used in the estimation increases $(n \rightarrow \infty)$. However, the rate at which $h$ is approaching 0 is much slower such that $(n h \rightarrow \infty)$.

The bandwidth $h$. Eqs. (1) and (3) use different bandwidth values to capture the spread of the data on each dimension. This suggests using the same analysis of estimating the bandwidth for the case of univariate data on the marginal distribution of the data on each dimension. Typically, bandwidth setting should minimize the deviation between the true and the estimated densities. This deviation is measured by the Mean Integrated Square Error (MISE) 35.

Let $\mu_{k}(x), R(f)$ be defined as $\mu_{k}(x)=\int x^{k} K(x) d x$ and $R(f)=\int f^{2}(x) d x$. The MISE of the estimator using a bandwidth value $h$ is

$$
\operatorname{MISE}(h)=\int E[\hat{f}(x)-f(x)]^{2} d x
$$

which has the asymptotic expansion $\operatorname{MISE}(h)=\operatorname{AMISE}(h)+O\left(n^{-1}+h^{5}\right)$ under suitable regularity conditions on $K$ and $f$. The minimizer of the $\operatorname{AMISE}(h)=$ $\frac{1}{n h} R(K)+h^{4}\left(\frac{\mu_{2}(K)}{4}\right)^{2} R\left(f^{\prime \prime}\right)$ is considered a good approximation for the optimal 
bandwidth value, which can be estimated as

$$
\hat{h}=\left(\frac{R(K)}{\mu_{2}^{2}(K) R\left(f^{\prime \prime}\right) n}\right)^{\frac{1}{5}} .
$$

However, this minimizer cannot be computed as it depends on the unknown density $f$.

Many methods have been introduced to estimate $R\left(f^{\prime \prime}\right)$ in Eq. (5). The normal rule 20] is the most popular method for estimating the bandwidth, which assumes the unknown density $f$ as a normal density and scales it according to the sample standard deviation. The bandwidth value selected using the normal rule is computed as:

$$
\hat{h}=c \hat{\sigma} n^{-1 / 5},
$$

where $c$ is a constant that depends on the used kernel function $K, \hat{\sigma}$ is the sample standard deviation and $n$ is the number of kernels. This method is computationally efficient but it does not work well when the density deviates significantly from normality. Other methods based on cross-validation have been proposed in the literature 36 37. These methods require performing density estimation for each candidate of the bandwidth values, which multiplies the computational cost by another factor equal to the number of candidates.

Plug-in methods [38 39] estimate an approximation of $R\left(f^{\prime \prime}\right)$ and plug it in Eq. (5) to compute the optimal bandwidth. Estimating $R\left(f^{\prime \prime}\right)$ requires also making assumptions about the density function but it becomes more accurate than using the normal rule. Sheather and Jones [37] estimate $R\left(f^{\prime \prime}\right)$ by estimating $f^{(4)}$, which in turn is estimated using $R\left(f^{(6)}\right)$. The value $R\left(f^{(6)}\right)$ is computed by assuming that $f^{(8)}$ is the eighth derivative of a normal density. After estimating $R\left(f^{(6)}\right)$, a backward substitution is performed to estimate $R\left(f^{\prime \prime}\right)$. Shimazaki and Shinomoto [4] assume that the true density follows a Poisson distribution and use Estimation-Maximization (EM) method to find the optimal bandwidth value. The method requires estimating the density for each estimation step of the EM optimization procedure, which will be very expensive in the case of streaming data where the density is changing dynamically and the bandwidth value needs to be estimated frequently.

In the case of multidimensional data, most of the bandwidth selection methods either consider a fixed bandwidth value for all the dimensions [1341] or use the marginal distribution to estimate the bandwidth on each dimension [14 20]. KDE fails to capture densities of arbitrary shapes when using the same bandwidth value for all dimensions. Methods with a different bandwidth value for each dimension rely on the marginal distribution of the data on that dimension.

The KDE-Track introduced next minimizes the effect of the normality assumption of $f$ by using the data samples to estimate $f^{\prime \prime}$. The numerical integration technique is then used to compute $R\left(f^{\prime \prime}\right)$, which is plugged in Eq. (5) to estimate the bandwidths. 


\subsection{KDE-Track Method}

KDE-Track models the distribution of the streaming data as a grid of resampling points and their corresponding estimated density values. For example in the 2dim sample space, let $\mathcal{U}^{1}=\left\{u_{0}^{1}, u_{1}^{1}, \ldots, u_{U^{1}-1}^{1}\right\}$ and $\mathcal{U}^{2}=\left\{u_{0}^{2}, u_{1}^{2}, \ldots, u_{U^{2}-1}^{2}\right\}$ be the set of points that discretize the range of the data on the first and the second dimensions, respectively. The KDE-Track model $\mathcal{M}$ is defined as the set of the grid points from $\mathcal{U}^{1} \times \mathcal{U}^{2}$ with their estimated densities. That is, $\mathcal{M}=\left\{M_{0}, M_{1}, \ldots, M_{q-1}\right\}$, where $q=U^{1} U^{2}$ is the number of the resampling points and $M_{s}$ is an ordered pair representing a grid point and its estimated $\operatorname{PDF}\left(M_{s}=\left(\boldsymbol{m}_{s}, \hat{f}\left(\boldsymbol{m}_{s}\right)\right)\right)$. Here $\boldsymbol{m}_{s}=\left(u_{k}^{1}, u_{l}^{2}\right) \in \mathcal{U}^{1} \times \mathcal{U}^{2}$ is the $s$-th resampling point with $l, k$ being the quotient and the remainder of the division of $s$ by $U^{1}$ and $\hat{f}\left(\boldsymbol{m}_{s}\right)$ is the density estimated using KDE at $\boldsymbol{m}_{s}$.

\section{Density estimation by interpolation.}

For a data sample $\boldsymbol{a}$ in 2-dim, the PDF at $\boldsymbol{a}$ can be efficiently estimated by bilinear interpolation of the resampling points, following two steps:

(1) fetch the estimated PDF values at resampling points $\boldsymbol{m}_{s 1}, \boldsymbol{m}_{s 1+1}, \boldsymbol{m}_{s 2}$ and $\boldsymbol{m}_{s 2+1}$ that surround the point $\boldsymbol{a}$ (as in Figure 11). Let $y^{(i)}$ be the projection of vector $\boldsymbol{y}$ on $i$-axis, then $m_{s 1}^{(1)}=m_{s 2}^{(1)} \leq a^{(1)}<m_{s 1+1}^{(1)}=m_{s 2+1}^{(1)}$ and $m_{s 1}^{(2)}=m_{s 1+1}^{(2)} \leq a^{(2)}<m_{s 2}^{(2)}=m_{s 2+1}^{(2)}$. Then linearly interpolate the density at $\boldsymbol{m}_{s 1}, \boldsymbol{m}_{s 1+1}$ to estimate the density at $\boldsymbol{r}_{s 1}$ and interpolate the density at $\boldsymbol{m}_{s 2}, \boldsymbol{m}_{s 2+1}$ to compute the density at $\boldsymbol{r}_{s 2}$;

(2) estimate the density at $\boldsymbol{a}$ by interpolating the density at $\boldsymbol{r}_{s 1}, \boldsymbol{r}_{s 2}$.

Let $D(\boldsymbol{b}, \boldsymbol{c})$ be the Euclidean distance between $\boldsymbol{b}$ and $\boldsymbol{c}$. The density at $\boldsymbol{a}$ will be computed as follows:

$$
\tilde{f}(\boldsymbol{a})=\frac{D\left(\boldsymbol{a}, \boldsymbol{r}_{s 2}\right) \tilde{f}\left(\boldsymbol{r}_{s 1}\right)+D\left(\boldsymbol{r}_{s 1}, \boldsymbol{a}\right) \tilde{f}\left(\boldsymbol{r}_{s 2}\right)}{D\left(\boldsymbol{r}_{s 1}, \boldsymbol{r}_{s 2}\right)},
$$

where

$$
\tilde{f}\left(\boldsymbol{r}_{s 1}\right)=\frac{D\left(\boldsymbol{r}_{s 1}, \boldsymbol{m}_{s 1+1}\right) \hat{f}\left(\boldsymbol{m}_{s 1}\right)+D\left(\boldsymbol{m}_{s 1}, \boldsymbol{r}_{s 1}\right) \hat{f}\left(\boldsymbol{m}_{s 1+1}\right)}{D\left(\boldsymbol{m}_{s 1}, \boldsymbol{m}_{s 1+1}\right)},
$$

and

$$
\tilde{f}\left(\boldsymbol{r}_{s 2}\right)=\frac{D\left(\boldsymbol{r}_{s 2}, \boldsymbol{m}_{s 2+1}\right) \hat{f}\left(\boldsymbol{m}_{s 2}\right)+D\left(\boldsymbol{m}_{s 2}, \boldsymbol{r}_{s 2}\right) \hat{f}\left(\boldsymbol{m}_{s 2+1}\right)}{D\left(\boldsymbol{m}_{s 2}, \boldsymbol{m}_{s 2+1}\right)} .
$$

KDE interpolation is efficient as it stores only the function at the resampling points whose total number is in the constant order and is small compared to the stream size. The running time for estimating the PDF for all $n$ arriving data samples will be in $O(n|\mathcal{M}|)$.

Error analysis. Three types of errors may be introduced by KDE-Track: the estimation error inherited from KDE, the interpolation error and the rounding error. Since rounding error (occurring when infinite number of digits after the decimal point are squeezed in a finite number of bits) is machine dependent, we focus on the interpolation error and propose an adaptive resampling model to 


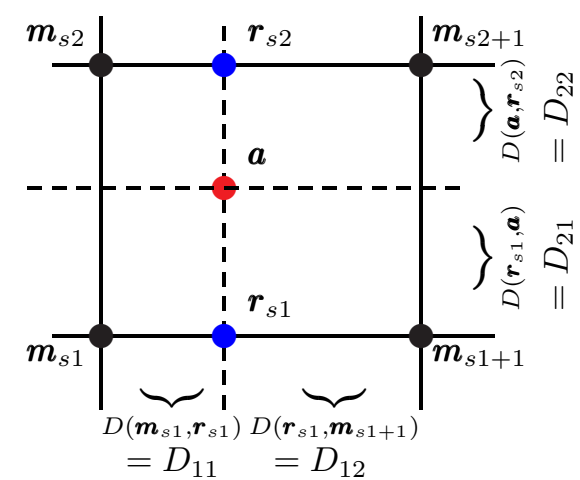

Fig. 1: Computing the density at $\boldsymbol{a}$ by interpolation given the KDE estimation $\hat{f}$ at $\boldsymbol{m}_{s 1}, \boldsymbol{m}_{s 1+1}, \boldsymbol{m}_{s 2}$ and $\boldsymbol{m}_{s 2+1}$.

minimize this type of error. The error inherited from KDE will be minimized by selecting the optimal bandwidth values for the KDE.

In [7, we studied the interpolation error for the case of univariate data. Let $\hat{f}(a)$ and $\tilde{f}(a)$ be the estimated PDFs using the traditional KDE and the KDETrack, respectively, and $D_{m}$ be the maximum distance between two consecutive resampling points. The error is: $\tilde{f}(a)-\hat{f}(a)=\frac{\left\{D_{m}\right\}^{2}}{8} \hat{f}^{\prime \prime}(a)+O_{p}\left(\left\{D_{m}\right\}^{3}\right)$. The interpolation error will increase in the case of multi-dimensional data. In 18, we derived the interpolation error in 2-dimension as $\tilde{f}(\boldsymbol{a})-\hat{f}(\boldsymbol{a})=\frac{D_{m}^{2}}{8}\left\{\hat{f}_{x_{1} x_{1}}(\boldsymbol{a})+\right.$ $\left.\hat{f}_{x_{2} x_{2}}(\boldsymbol{a})\right\}+O_{p}\left(\left\{D_{m}\right\}^{3}\right)$, where $D_{m}$ is the distance between two consecutive resampling points in 2-dimension. Note that the term $O_{p}\left(D_{m}^{3}\right)$ includes also the terms with higher order derivatives. When using the Epanechnikov kernel function, the second partial derivative will be constant and partial derivatives of higher order will be zeros.

Adaptive resampling model. From the above error analysis, we know that the accuracy of the linear interpolation depends on 1) the distance between two adjacent resampling points; and 2) the second derivative of the density function. To minimize the error while keeping the number of resampling points within a reasonable margin, more resampling points can be added in the regions where the density function has high curvature, as shown in Figure 2. By contrast, in the regions with approximately linear function, less resampling points are used.

In multi-dimensional data streams, the distribution is usually spatial, nonuniform and dynamic. Therefore, high resolution with sufficient resampling points is required 1) in dense areas with high PDF values to catch the details; and 2) in sensitive areas which are the boundary between dense and sparse to catch dynamic changes. Adaptive resampling meets the requirement perfectly. 


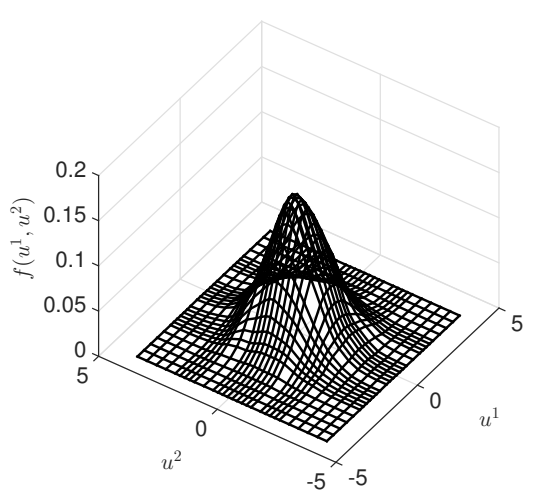

Fig. 2: Example of adaptive resampling: more resampling points are used in regions with high curvature of the function.

Bandwidth selection. KDE-Track uses different bandwidth values for each dimension. The marginal distribution of the data is used to compute the bandwidth value on that dimension. From Eq. (5), the bandwidth value on dimension $i$ can be computed as

$$
\hat{h}_{i}=\left(\frac{R(K)}{\mu_{2}^{2}(K) R\left(f_{i}^{\prime \prime}\right) n}\right)^{\frac{1}{5}},
$$

where $f_{i}^{\prime \prime}$ is the second derivative of the density function on the $i$-th dimension. Initially, a pilot bandwidth is estimated using the normal rule $\tilde{h}_{i}=c \hat{\sigma}_{i} n^{-1 / 5}$, where $c$ is a constant that depends on the kernel function $K$ and $\hat{\sigma}_{i}$ is the standard deviation of the projection of the data on axis $i$. This pilot bandwidth is used to estimate the second derivative of the marginal distribution as

$$
\hat{f}_{i}^{\prime \prime}(x)=\frac{1}{n \tilde{h}_{i}} \sum_{j=1}^{n} K^{\prime \prime}\left(\frac{x-x_{j}}{\tilde{h}_{i}}\right)
$$

In this case, $\hat{f}_{i}^{\prime \prime}$ will be a better approximation of $f_{i}^{\prime \prime}$ than considering $f_{i}$ to be a normal density. Using KDE-Track on the one dimensional data will speedup the computation of $R\left(\hat{f}_{i}^{\prime \prime}\right)$ and $\hat{h}_{i}$. Since the distribution of the data will change over time with the arrival of new samples from the stream, the bandwidth values $\hat{h}_{i}$ should be updated accordingly to represent the variation of the data along the different dimensions. Using KDE-Track will also allow for updating the values of $\hat{h}_{i}$ online and efficiently.

In this way, the estimated $\hat{f}_{i}^{\prime \prime}$ will serve two roles. First, it is used to approximate $R\left(f_{i}^{\prime \prime}\right)$ to compute the bandwidth value. Second, it is used as a more accurate indicator of the high curvature of the density function's curve, which facilitates the adaptive resampling in KDE-Track for obtaining more accurate estimation as we will discuss in the following subsections. 


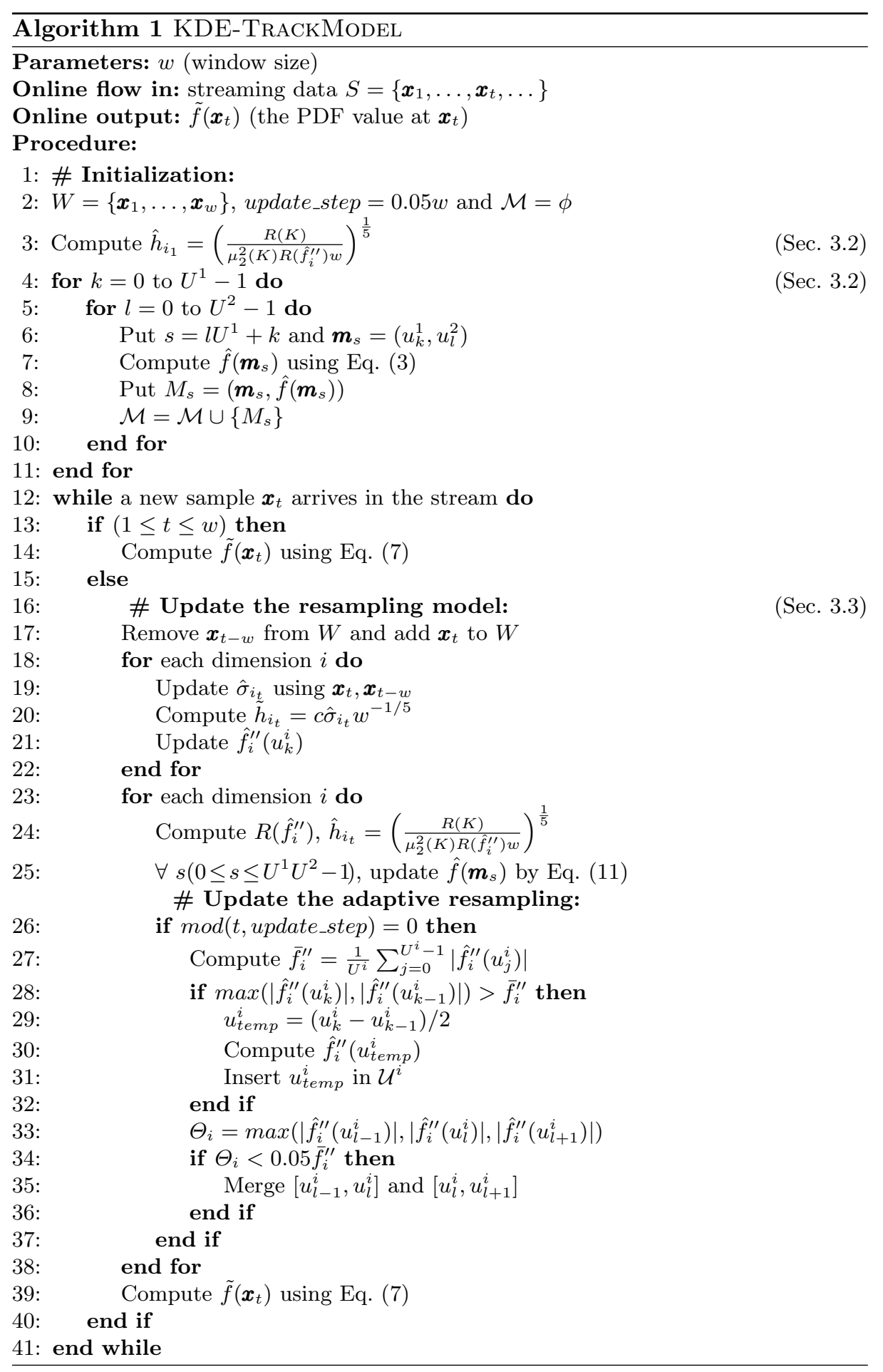




\subsection{KDE-Track Implementation}

Estimating the density for each incoming data samples using KDE-Track requires access to four resampling points only as discussed in Section 3.2 The key step is thus the maintenance of the resampling model (resampling points and their PDF values). Algorithm 1 shows the maintenance of KDE-Track's model and using it for online density estimation. Lines start with the \# sign represent comments.

Initializing the resampling model. The resampling model is initialized by the beginning part of streaming data, e.g., the first 5000 point: ${ }^{4}$. The resampling points, $\boldsymbol{m}_{s}, s=1, \ldots, q$, are defined as the cartesian product of the set of equidistant points selected on each dimension within the range of initial points received so far ${ }^{5}$. The second derivative of the marginal density on each dimension is then estimated at the initial resampling points and used for selecting the bandwidth value. Moreover, the second derivative is used to add more resampling points in the regions with high curvature of the density. Using the estimated bandwidth value, the density values $\hat{f}\left(\boldsymbol{m}_{s}\right)$ of these resampling points are computed using the traditional KDE on the initial batch of points.

Estimation based on the resampling model. Once all $M_{i}$ have been initialized, the density at each arriving point $\boldsymbol{x}$ can be estimated. Due to the usage of interpolation method, the density at $\boldsymbol{x}$ can be estimated by 1) calculating, on each dimension, the index of one resampling point who and whose successive neighbor will contribute; 2) fetching the four resampling points in the cell surrounding $\boldsymbol{x}$ and their densities; and 3) computing $\tilde{f}(\boldsymbol{x})$ using Eq. (7).

Updating the resampling model. The resampling model is the basis of the density estimator. The resampling points and their PDF values should be updated after receiving a new data point. As discussed in Subsection 3.2 the resampling points are adaptively maintained according to the curvature of density function. An interval $\left[u_{j}^{i}, u_{j+1}^{i}\right]$ is divided into two equal subintervals when $\max \left\{\left|\hat{f}_{i}^{\prime \prime}\left(u_{j}^{i}\right)\right|,\left|\hat{f}_{i}^{\prime \prime}\left(u_{j+1}^{i}\right)\right|\right\}>\bar{f}_{i}^{\prime \prime}$, where $\bar{f}_{i}^{\prime \prime}=\frac{1}{U^{i}} \sum_{j=0}^{U^{i}-1}\left|\hat{f}_{i}^{\prime \prime}\left(u_{j}^{i}\right)\right|$ is the average of the second derivative absolute values. In this way, more resampling points are inserted in areas which are the boundary between dense and sparse or are dense with high peak values in density function. Two intervals $\left[u_{l-1}^{i}, u_{l}^{i}\right]$ and $\left[u_{l}^{i}, u_{l+1}^{i}\right]$ are merged to reduce the number of resampling points when the density function is close to linear, which means $\left|\hat{f}_{i}^{\prime \prime}\left(u_{l-1}^{i}\right)\right|,\left|\hat{f}_{i}^{\prime \prime}\left(u_{l}^{i}\right)\right|$ and $\left|\hat{f}_{i}^{\prime \prime}\left(u_{l+1}^{i}\right)\right|$ are close to zero (less than $0.05 \bar{f}_{i}^{\prime \prime}$ ). In sparse regions, the PDF values are close to zero and the function is almost linear so the intervals are also merged to reduce the number of resampling points.

\footnotetext{
${ }^{4}$ This first batch of data is used for initializing the resampling points and setting bandwidth values. KDE-Track is not sensitive to how many points are used in this batch, as the resampling model and bandwidth are updated online with new arriving data after initialization.

5 The initial model is defined such that the distance between any two consecutive points on the $x_{i}$ axis is $\tilde{h}_{i_{1}}$, where $\tilde{h}_{i_{1}}$ is the pilot bandwidth estimated using the first batch of data points.
} 
When updating the densities of resampling points in the model $\mathcal{M}$, the evolution of the data distribution should be considered. Here, a sliding window strategy is used to catch the evolution over time. The window size $w$ is an application dependent parameter and can be set based on the arrival rate of the data samples and the time interval during which we need to estimate the dynamic density. The window size also controls the robustness of KDE-Track against noisy data where an isolated outlier will increase the height of the PDF curve by maximum $1 / w$, which will not be noticed. However, when a new pattern arrives, the new points will replace points in the sliding window from the old pattern and their contribution will be observed on the shape of the density function after receiving a reasonable number of data points from the new pattern. Let $n_{t}$ denote the number of points that have been received until time $t$. Due to the difference between $w$ and $n_{t}$, there are two different scenarios when updating the model $\mathcal{M}$, more specifically, updating the bandwidths and density $\hat{f}\left(\boldsymbol{m}_{s}\right)$.

When $n_{t} \leq w$. The received points cannot fill the whole window. The pilot bandwidth value at time $t$ is calculated using all $n_{t}$ points by the formula $\tilde{h}_{i_{t}}=$ $c \hat{\sigma}_{i_{t}} n_{t}^{-1 / 5}$, where $\hat{\sigma}_{i_{t}}^{2}$ is the sample variance of the received data samples projected on the $i$-th axis calculated as $\hat{\sigma}_{i t}^{2}=\frac{1}{n_{t}-1}\left\{\sum_{j=1}^{n_{t}} x_{i j}^{2}-\frac{1}{n_{t}}\left(\sum_{j=1}^{n_{t}} x_{i j}\right)^{2}\right\}, i \in$ $\{1,2\}$ [42, which can be updated with a constant time at each $t$. The pilot bandwidth is used to update the estimation of the second derivative of the data marginal distribution on dimension $i$. The roughness $R\left(f_{i}^{\prime \prime}\right)$ is then computed to estimate the bandwidth value on that dimension.

After receiving a point $\boldsymbol{x}_{t}$, the density at a resampling point $\boldsymbol{m}_{s}$ at time $t$ is updated using sample-point estimator 43 .

$$
\hat{f}_{t}\left(\boldsymbol{m}_{s}\right)=\frac{n_{t}-1}{n_{t}} \hat{f}_{t-1}\left(\boldsymbol{m}_{s}\right)+\frac{1}{n_{t} \hat{h}_{1_{t}} \hat{h}_{2_{t}}} K_{\hat{h}}\left(\boldsymbol{m}_{s}, \boldsymbol{x}_{t}\right),
$$

where $K_{\hat{h}}$ is defined in Eq. (3). It is straightforward to show that the updated density $\hat{f}_{t}\left(\boldsymbol{m}_{s}\right)$ is a good approximation to the estimated density using all the $n_{t}$ points. In particular, since $\hat{f}_{t}(\boldsymbol{x})=\frac{1}{n_{t}} \sum_{j=1}^{t} \frac{1}{\hat{h}_{1_{j}} \hat{h}_{2_{j}}} K_{\hat{h}_{j}}\left(\boldsymbol{x}, \boldsymbol{x}_{j}\right) \geq 0$ and $\forall j$ the integration over $\boldsymbol{x}$ of $\frac{1}{\hat{h}_{1_{j}} \hat{h}_{2_{j}}} K_{\hat{h}_{j}}\left(\boldsymbol{x}, \boldsymbol{x}_{j}\right)=1$, averaging the integrations of the two terms in 10 results in 1 .

When $n_{t}>w$. In this case, the pilot bandwidth is calculated on the most recently received $w$ points inside the window as follows: $\hat{h}_{i_{t}}=c \hat{\sigma}_{i_{t}} w^{-1 / 5}$, where the sample variance $\hat{\sigma}_{i_{t}}^{2}$ of the projected data on the $i$-th dimension can be easily updated by $\hat{\sigma}_{i_{t}}^{2}=\frac{1}{w-1}\left(\sum_{j=t-w+1}^{t} x_{i j}^{2}-\frac{1}{w}\left(\sum_{j=t-w+1}^{t} x_{i j}\right)^{2}\right)$. The pilot bandwidth is used to update the estimation of the second derivative values and to compute the bandwidth $\hat{h}_{i_{t}}$, which is used to update the density function at the resampling points. The PDF values at the resampling points $\hat{f}_{t}\left(\boldsymbol{m}_{s}\right)$ are updated by absorbing the new arrived point $\boldsymbol{x}_{t}$ and deleting the old point that 
was moved out from the window:

$$
\hat{f}_{t}\left(\boldsymbol{m}_{s}\right)=\hat{f}_{t-1}\left(\boldsymbol{m}_{s}\right)+\frac{K_{\hat{h}_{t}}\left(\boldsymbol{m}_{s}, \boldsymbol{x}_{t}\right)}{w \hat{h}_{1_{t}} \hat{h}_{2_{t}}}-\frac{K_{\hat{h}_{t}}\left(\boldsymbol{m}_{s}, \boldsymbol{x}_{t-w}\right)}{w \hat{h}_{1_{t-w}} \hat{h}_{2_{t-w}}} .
$$

The probabilistic properties of updated density function $\hat{f}_{t}(x)$ can be proved as:

1. $\hat{f}_{t}(\boldsymbol{x}) \geq 0, \forall \boldsymbol{x}$, due to the fact that $\hat{f}_{t}(\boldsymbol{x})=\frac{1}{w} \sum_{j=t-w+1}^{t} \frac{1}{h_{1_{j}} \hat{h}_{2_{j}}} K_{\hat{h}_{j}}\left(\boldsymbol{x}, \boldsymbol{x}_{j}\right)$ is a summation of nonnegative terms;

2. the integration $\int_{-\infty}^{\infty} \hat{f}_{t}(\boldsymbol{x}) d x_{1} d x_{2}=1$. Since for any $j$ the integration of $\left(\hat{h}_{1_{j}} \hat{h}_{2_{j}}\right)^{-1} K_{\hat{h}_{j}}\left(\boldsymbol{x}, \boldsymbol{x}_{j}\right)=1$, averaging $w$ terms will also be 1 .

Time and Space Complexity Analysis. Based on the discussion earlier in this section, the time complexity of estimating the density for a new incoming data point is $O\left(U^{1}+U^{2}\right)$, where $U^{1}$ and $U^{2}$ were given in the beginning of Section 3.2. They are independent of the number of points that have been received from the data stream. Updating the model when receiving a new point requires computing time linear to the total number of the resampling points $|\mathcal{M}|$, since all the function values at the resampling points are updated. The overall time complexity of processing each arriving point is linear to the model size, which is usually a limited small number. The time required for online density estimation of a data stream with $n$ points is $O(n|\mathcal{M}|)$, which linearly increases with the number of received points from the stream.

Bandwidth selection requires maintaining a one dimensional KDE-Track model on each dimension. Since $U^{1} \times U^{2}=|\mathcal{M}|$ and $U^{1}, U^{2} \geq 1$, the total number of resampling points in both models of the one dimensional KDE-Track is $U^{1}+U^{2} \leq|\mathcal{M}+1|$, which will increase only the constant in the KDE-Track's time complexity formula. Thus, the KDE-Track time complexity is $O(n|\mathcal{M}|)=$ $O\left(n \times U^{1} \times U^{2}\right)$.

During the online density estimation process, KDE-Track keeps the resampling model $\mathcal{M}$ and the points in the sliding window in memory. Therefore, the memory usage is $|\mathcal{M}| w=U^{1} \times U^{2} \times w$. Note that the model size $|\mathcal{M}|$ changes upon the distribution variation in data streams due to merge/split operations in adaptive resampling.

Multidimensions. Extending the two dimensional KDE-Track to higher dimensions is straightforward. The same technique can be used for selecting the bandwidth using the marginal distribution of the data on each dimension. The KDE-Track model for estimating the density of $d$-dimensional data can be constructed as follows:

1) discretize the range of the data on the $i$-th dimension, with $1 \leq i \leq d$, using a set of points $\left.\mathcal{U}^{i} ; 2\right)$ define the set of resampling points as the cartesian product $\mathcal{U}^{1} \times \mathcal{U}^{2} \times \cdots \times \mathcal{U}^{d}$; and 3) estimate the density function values at the set of resampling points and store them with their estimated density in the model $\mathcal{M}$.

The product kernel defined in Eq. (3) will be

$$
K_{h}\left(\boldsymbol{x}, \boldsymbol{x}_{j}\right)=\prod_{i=1}^{d}\left\{\frac{1}{h_{i}} K\left(\frac{x_{i}-x_{j i}}{h_{i}}\right)\right\} .
$$


Some researchers avoid using the product kernel for the case of high dimensional data and replace it with an orientation-invariant kernel function

$$
K_{h}\left(\boldsymbol{x}, \boldsymbol{x}_{j}\right)=\frac{1}{h} K\left(\frac{\left\|\boldsymbol{x}-\boldsymbol{x}_{j}\right\|}{h}\right),
$$

which may not be able to estimate densities with arbitrary shapes as it assumes equal variance values of the data on each dimension.

The KDE-Track's time complexity will remain linear in the size of the stream $O(n|\mathcal{M}|)$, but the constant in the complexity formula will increase according to the number of dimensions since $|\mathcal{M}|=\left|\mathcal{U}^{1}\right| \times\left|\mathcal{U}^{2}\right| \times \cdots \times\left|\mathcal{U}^{d}\right|$. Let $\bar{U}=\frac{1}{d} \sum_{i=1}^{d}\left|\mathcal{U}^{i}\right|$ then $|\mathcal{M}|=\bar{U}^{d}$ and the time complexity of KDE-Track will be $O\left(n \bar{U}^{d}\right)$.

The interpolation error for the case of $d$-dimensional data can be bounded as follows: let $D_{m}^{i}$ be the maximum distance between the resampling points in dimension $i, i \in\{1,2, \ldots, d\}$ and $D_{m}=\max \left\{D_{m}^{i}, 1 \leq i \leq d\right\}$. Then we can show using mathematical induction on $d$ that

$$
\tilde{f}(\boldsymbol{a})=\hat{f}(\boldsymbol{a})+\frac{D_{m}^{2}}{8}\left\{\sum_{i=1}^{d} \hat{f}_{x_{i} x_{i}}(\boldsymbol{a})\right\}+O_{p}\left(D_{m}^{3}\right) .
$$

The error is reducible by including more resampling points in certain regions. However, using KDE-Track for estimating high dimensional density might become impractical as the data become sparse and the estimation error will be large. Besides, applications that rely on visualizing the density functions will not benefit from estimating the density for high dimensional data as the density cannot be visualized in the case of data streams with more than three dimensions.

\section{Density Estimation Performance Evaluation}

This section evaluates the most popular density estimators, including 1) the traditional KDE [20] defined in Eq. [1]; 2) the FFT-KDE [20|19], which deploys FFT to convolve a very fine histogram of the data with a kernel function to produce a continuous density function; 3) the Cluster Kernels (CK) [10, which maintains a specific number of kernels by merging similar kernels; 4) SOMKE 12, which employs SOM to cluster the data into a specific number of clusters and uses the centroids of the clusters as the set of kernels; and 5) KDE-Track [18] presented in Section 3.2 .

\subsection{Estimation accuracy on synthetic data}

Datasets. The one-dim stream (S1D) was generated by extracting data samples from the fifteen densities suggested by Marron and Wand 44 and presented in Figure 3 . The stream is constructed by extracting $3 \times 10^{4}$ data samples from each density and concatenating the batches to get one stream of $4.5 \times 10^{5}$ data samples. 

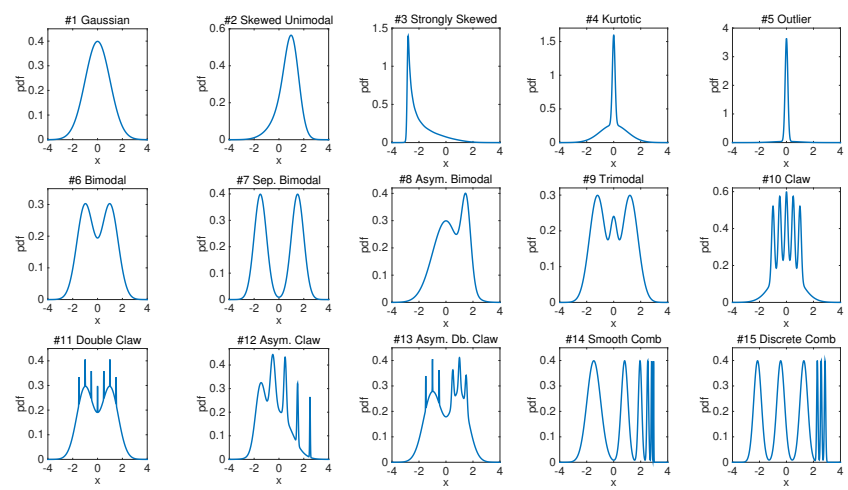

Fig. 3: The fifteen densities recommended by Marron and Wand 44 to evaluate univariate density estimators.

The two-dim data stream (S2D) is generated by extracting data segments of size $10^{5}$ from the seven densities presented in Figure 4. The total size of the stream is $7 \times 10^{5}$ data samples. These streams are selected because they contain challenging densities that are hard to be estimated accurately. Using batches of the same size is to simplify the calculation of the true density only.
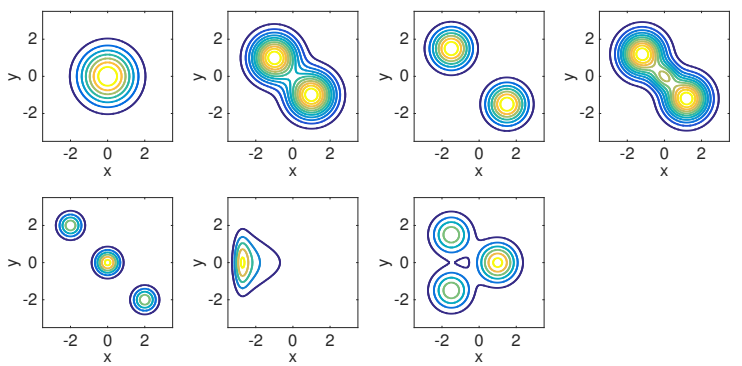

Fig. 4: The contours of the densities used to construct the 2D data stream that is used to evaluate the density estimators.

Estimation accuracy. Figures 5 shows the MAE and the $l_{\infty}$ error incurred by the evaluated methods when estimating the density of S1D (Subfigures a, b) and S2D (Subfigures c, d). The MAE measures how the estimated density curve fits the curve of the true density, while the $l_{\infty}$ measures the maximum variation between the true and the estimated curves. The error is computed by defining a set of checkpoints with the step of 1000. For each estimation method, at each checkpoint an evaluation set $E=\left\{\boldsymbol{e}_{1}, \ldots, \boldsymbol{e}_{1000}\right\}$ of 1000 samples is generated from the same distribution of the data in the sliding window. The true and 


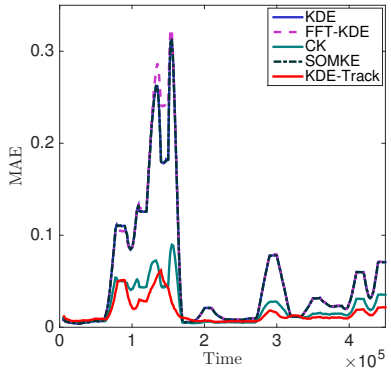

(a) MAE of S1D

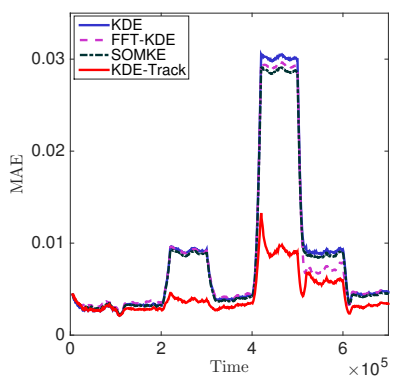

(c) MAE of S2D

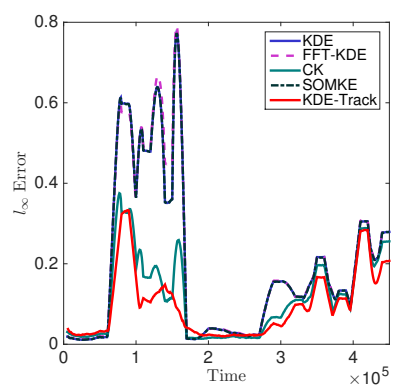

(b) $l_{\infty}$ error of S1D

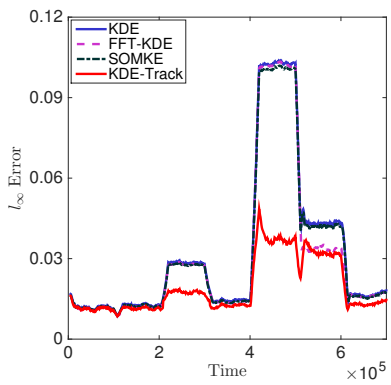

(d) $l_{\infty}$ error of S2D

Fig. 5: The MAE and the $l_{\infty}$ error incurred by the different density estimators when estimating the density of the S1D and S2D stream. The window size is $2 \times 10^{4}$.

estimated density values of the evaluation points are then compared to compute the MAE and the $l_{\infty}$ error. The window size is set to $2 \times 10^{4}$ data samples.

The CK and FFT-KDE methods are not designed to capture the dynamic density of the data streams using the sliding window approach. To adapt these methods with sliding windows, we rebuild their model at each evaluation checkpoint by deleting the old model and creating a new model using the data samples in the current window. This adaptation preserves the estimation accuracy of the methods. However, the CK method is shown to be impractical for online density estimation due to its high computational cost, as we will show later. SOMKE is adapted for the case of sliding window by dividing the sliding window into batches of 1000 samples. At each evaluation checkpoint, the kernels that represent the removed batch out of the sliding window are deleted and replaced by the kernels that represent the most recent batch added to the sliding window. Notice that CK is not evaluated on S2D data as CK is proposed to estimate the density for univariate data only.

Selecting the bandwidth values for each estimator is done using the same settings as in the references. All the baseline methods use the normal rule because of its efficiency, except the CK method which uses the Epanechnikov kernel function with a recommended constant $c=1.06$. This setting enables CK to perform well when densities have high peaks and are multimodal. KDE-Track uses its 


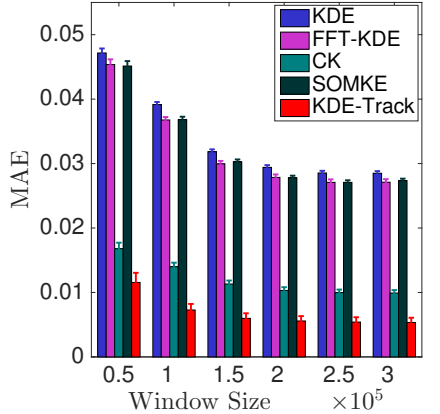

(a) S1D

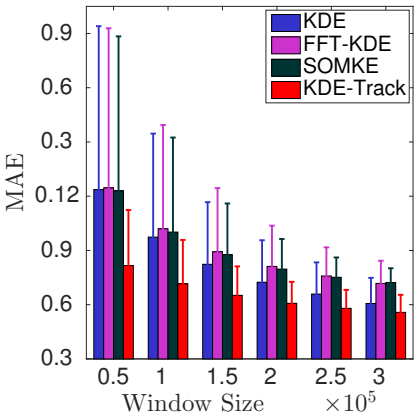

(b) S2D

Fig. 6: The MAE incurred by the different density estimators when estimating the density of the S1D stream (a) and S2D stream (b). The window size varies from $5 \times 10^{4}$ to $3 \times 10^{5}$.

own method for setting the bandwidths, i.e., estimating the roughness of the second derivative $R\left(f^{\prime \prime}\right)$ and plugging it in Eq. (5), which increases its accuracy significantly. The results show that KDE-Track has the best performance (the smallest error). The high accuracy obtained by KDE-Track is mainly because of the accurate bandwidth selection method.

Figure 6 shows the estimation error in terms of MAE for the different estimators when estimating the densities of S1D and S2D with different sliding windows. The sliding window's size changes from $5 \times 10^{4}$ to $3 \times 10^{5}$. For large windows, the density estimation becomes more accurate, which is reflected by smaller MAE values. However, the decrease in the MAE is not as expected because the larger windows include data from different densities, which complicates the density estimation process. KDE-Track is shown to have the most accurate results. KDE, FFT-KDE and SOMKE have comparable results. In addition to the MAE, Figure 6 shows the standard deviation for the sensitivity analysis of the window size, where KDE-Track is the most accurate (with the lowest error) and most stable (with the smallest standard deviation in error), especially in the S2D streams.

\subsection{Computational time cost and space usage}

Other important factors in the success of an online density estimator is its running time and space usage, as streaming data arrive fast and have unlimited size. Since we are estimating the dynamic density, which will be better represented by the most recent samples, all the methods are modified to use sliding window technique. This technique requires storing the samples in the sliding window in the memory either for using them to estimate the density as in KDE or to update the density estimator's model as in CK, FFT-KDE, SOMKE and KDE-Track. Hence, all the methods have comparable space complexity, which is linear in the window size. 


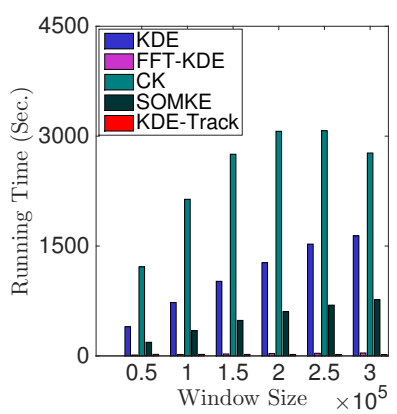

(a) S1D

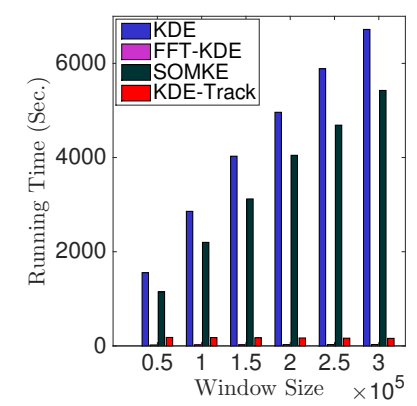

(b) S2D

Fig. 7: The running time of the different density estimators when estimating the density of the S1D (a) and S2D (b) streams. The window size varies from $5 \times 10^{4}$ to $3 \times 10^{5}$.

The time complexity of KDE-Track, as discussed in Subsection 3.3 , is $O(n|\mathcal{M}|)$. Estimating the density using KDE at any given data sample requires scanning the sliding window. Therefore, the time complexity of KDE when used for online density estimation is $O(n w)$, where $w$ is the window size. The time complexity of CK is controlled by two main steps: 1) model reconstruction, which is performed at each evaluation checkpoint and has a complexity of $O(w) ; 2)$ density estimation at any sample of the evaluation points, which has a constant time complexity. Thus, using CK for online density estimation will have a complexity of $O(n w)$. However, the model's reconstruction step of CK is more expensive than the density estimation using all the kernels in KDE. It is expected that CK will be more timely efficient if the data is stationary and the model is updated online without reconstruction.

FFT-KDE also has two main steps: 1) model reconstruction, which involves updating the histogram after receiving a new data sample and convolving the histogram with kernel function; and 2) density estimation of the evaluation samples. The first step requires $O(B \log B)$, where $B$ is the number of bins in the histogram, and the second step has a constant time complexity. The time complexity is thus $O(n B \log B)$.

The SOMKE model is built by training the SOM neurons with the current window which has a time complexity of $O(w)$. Estimating the density at the evaluation samples using the trained SOM neurons has a constant time complexity. The method's time complexity is then $O(n w)$, where the constant in the complexity formula is smaller than that for KDE and CK. Figure 7 shows the running time for using the density estimators for online density estimation of S1D and S2D streams. The results in the figure confirm our analysi ${ }^{6}$, KDETrack and FFT-KDE are most efficient with very small running time, which is not affected by the size of $w$.

\footnotetext{
${ }^{6}$ All implementations were coded by $\mathrm{C} / \mathrm{C}++$ and run on Intel $2.5 \mathrm{GHz}$ Dual-Core
} PC with 4 GB memory. 


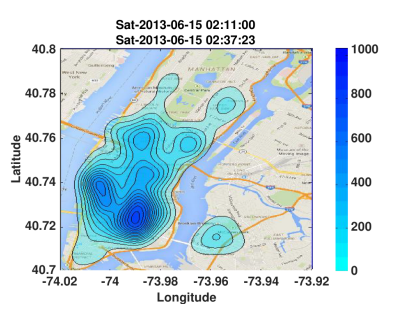

(a) weekend

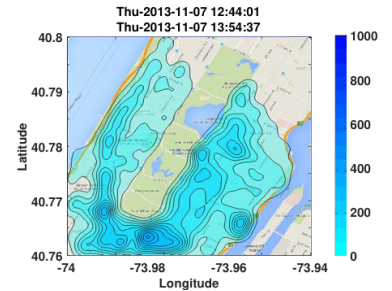

(d) working hours

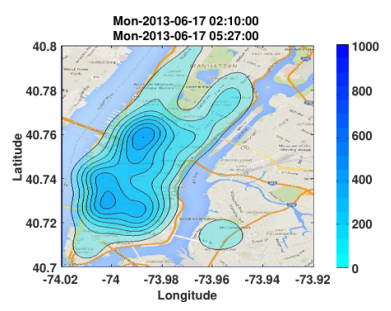

(b) working day Thu-2013-11-07 21:48:47
Thu-2013-11-07 22:55:00

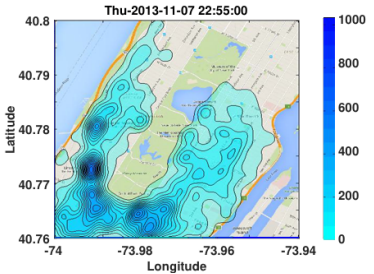

(e) night

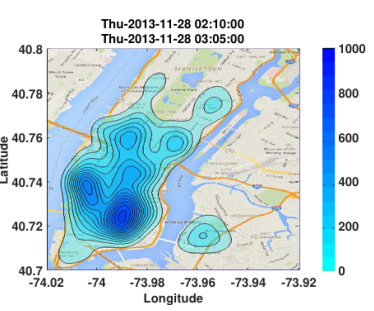

(c) Thanksgiving Fri-2013-11-08 00:33:22

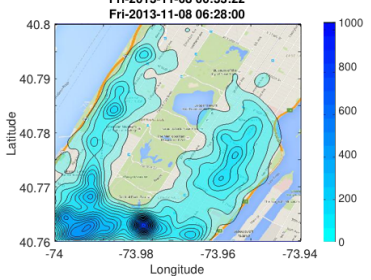

(f) after midnight

Fig. 8: The density estimated using New York Taxi trips data for different time intervals.

\section{Applications}

In this section, we apply the estimated dynamic density to two different application problems: Taxi traffic real-time visualization and unsupervised online change detection.

\subsection{Visualizing the Taxi Traffic data}

Visualizing the density function in real time can help service planners in monitoring the density of required services and forwarding more service providers to regions that demand more services at a specific time. For example, monitoring the density of taxi pickup data can tell the planners of taxi companies to forward more taxicabs to a specific region of the city. In this subsection, we visualize the dynamic traffic distribution in the New York Taxi trips dataset 7 . The dataset is freely available and contains records of trips that include pickup time, longitude and latitude of the pickup and drop off location, etc. We are mainly interested in the pickup time and location. Figure 8 shows the density estimated using the pickup location with window size of $10^{4}$, where the data records are sorted according to their pickup time. The first three subfigures show the pickup events occur in the early morning of a weekend day (subfigure (a)), of a regular working day (subfigure (b)) and of a national holiday (subfigure (c)). These subfigures show more pickup events during the weekends and holidays than during regular working days in the Greenwich and the east villages where there are many restaurants and nightclubs. The frequency of pickup events also increases during

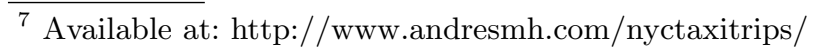


the weekends as it took less than 30 minutes to record $10^{4}$ events in a weekend but more than 3 hours in the early morning of a working day. More taxicabs are thus suggested in that region on similar events to satisfy the high demand.

Interesting patterns of community behavior can also be found in a regular working day. Subfigures 8 (d), (e), and (f) show the pickup events on November 7 and 8, 2013 at different time intervals. The pickup events during the working hours (subfigure (d)) show close to uniform distribution within the area around the central park. Subfigure 8 (e) shows high density at Lincoln center during the time interval 21:48 - 22:55, when a concert or other events may be over. After midnight, we can observe a small number of pickup events occurred as it took six hours to accumulate $10^{4}$ events with more pickup events occurred around Trump and Freedom towers.

\subsection{Online Change Detection}

Change detection in data streams refers to the problem of finding time points, where for each point, there exists a significant change in the current data distribution. A typical window-based solution is to extract a fixed $S_{1}$ (reference window) from streaming samples and to update an $S_{2}$ (test window) with newly arriving samples 2823 . Changes are then detected by measuring the difference between the distributions in $S_{1}$ and $S_{2}$.

Modeling the data distribution and selecting a comparison criterion are essential for change detection in data streams. However, density estimation of multidimensional data is difficult. It becomes less accurate and more computationally expensive with increasing dimensionality. In this subsection, we introduce a framework which applies Principal Component Analysis (PCA) to project the multidimensional data from the stream on the principal components to obtain multiple 1D data streams. Density estimation, distribution comparison, and change-score calculations can then be conducted in parallel on those 1D data streams. Compared with projecting the data on the original coordinates (i.e., using the original variables), projecting on PCs has the following advantages: 1) it allows the detection of changes in data correlations, which cannot be detected in the original individual variables; 2) it guarantees that any changes in the original variables are reflected in PC projections; and 3) it reduces the computation cost by discarding trivial PCs. Proofs can be found in [33].

Change Detection Framework. The framework is given in Algorithm 2 , where $D_{M}$ denotes any divergence metric for comparing two distributions.

Setting Windows: Line 3 in Algorithm 2 sets the reference window $S_{1}$ to be the first $w$ samples arriving after the change point $t_{c}$. Intuitively, when a data distribution shifts to a new one, the reference window should be updated to represent the new distribution. This update also enables the detection of further changes. Line 8 sets the test window $S_{2}$ as a collection of $w$ samples after the reference window. This $S_{2}$ will slide along the data stream to include the newest $w$ samples. The setting of this parameter is usually left to the user to give them 
the ability to monitor the long/short term changes, depending on their interests and the application sensitivity against changes [24].

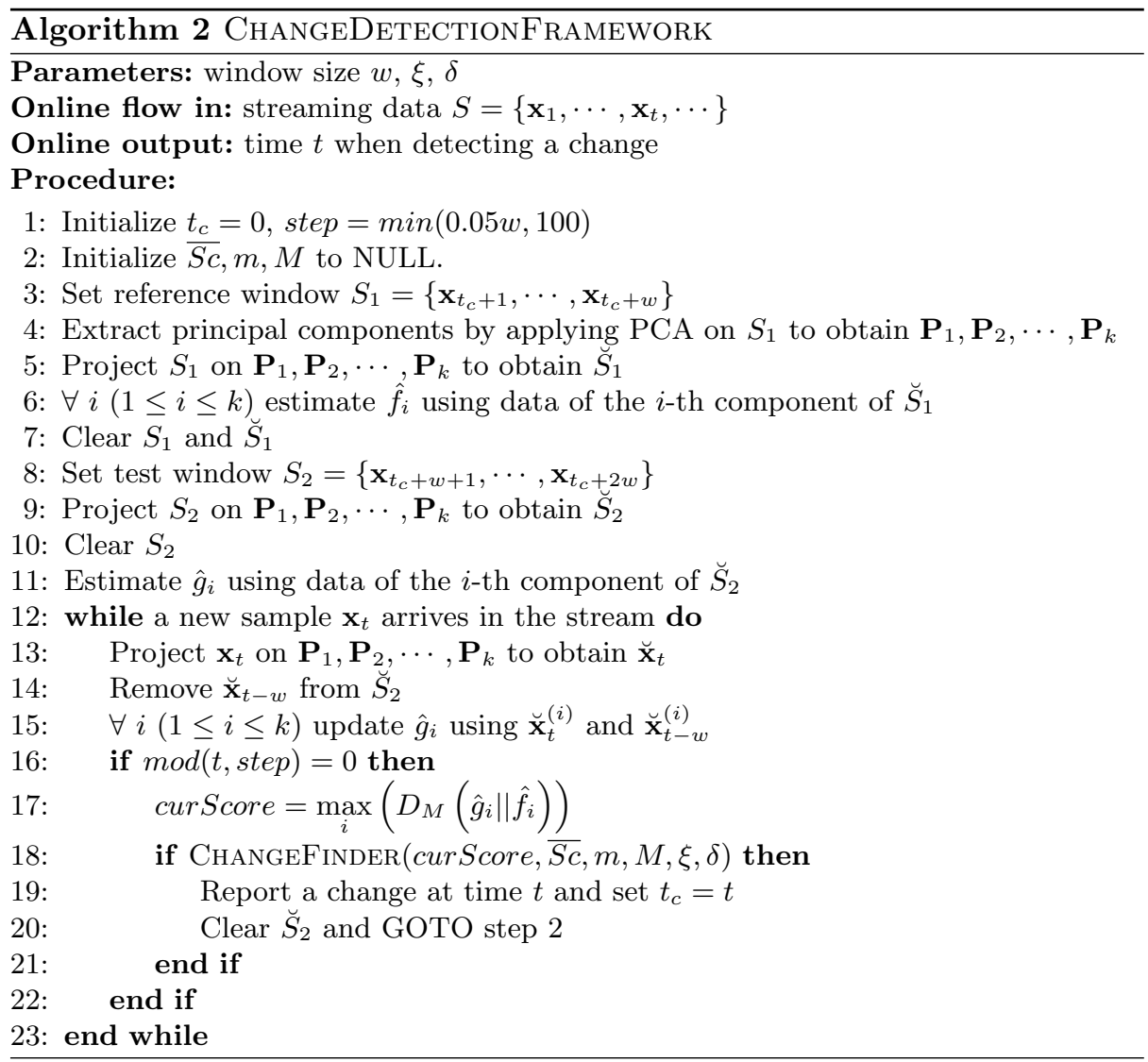

Projecting the Data: After receiving the first $w$ data samples in reference window $S_{1}$, PCA is applied to extract the principal components from $S_{1}$. The first $k$ PCs with the largest eigenvalues are selected if they account for $99.9 \%$ of data variance (i.e., $\sum_{i=1}^{k} \frac{\lambda_{i}}{\sum_{j=1}^{d} \lambda_{j}} \geq 0.999$ ). The data in the reference and test windows are then projected on these $k$ components. On each component, projections of the reference and test windows are compared and a change-score value is recorded. The maximum value among the $k$ change-score values is considered as the final change-score. Any new data sample is projected on the $k$ components and the density functions of the projection of the test window are updated and compared with the reference densities.

Estimating Density Functions: Because the change-score is used directly to trigger change alarms, PDFs for distribution comparison must be accurately and 
efficiently estimated. Here, KDE-Track is employed for estimating the density functions of the projected data of $S_{1}$ (line 6) and $S_{2}$ (line 11), as well as for updating the test densities when a new sample arrives (lines 13-15), due to its merits of both efficiency and accuracy. Note that the update at line 15 ensures that $\hat{g}_{i}$ is the current distribution of the $i$-th component in the data stream.

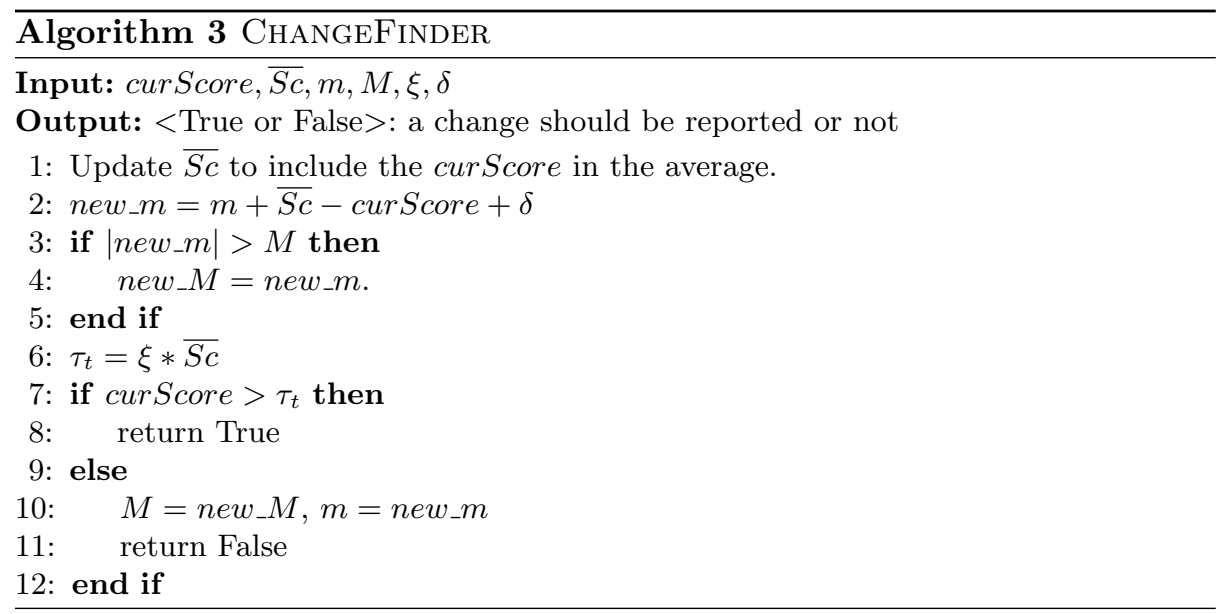

Computing the Change-Score Values: Change-scores are computed by using a divergence metric on two density functions $\hat{f}_{i}$ and $\hat{g}_{i}$ (line 17), which are updated upon the arrival of each sample. However, it is not necessary to compute change-score at each time step, as the change of a distribution cannot be observed after a single data sample. Therefore, to reduce unnecessary repeated comparisons that may increase the execution time noticeably, we compute change-scores every $\min (0.05 * w, 100)$ samples (line 16). This setting of checkpoints complies with the monitoring requirements of users. Monitoring short-term changes with a small $w$ needs frequent check points while monitoring long-term changes by setting a large $w$ allows bigger checkpoint intervals. The granularity can be adjusted by changing the $5 \%$ according to the users' needs.

Divergence metric $D_{M}\left(\hat{g}_{i} \| \hat{f}_{i}\right)$ is crucial for computing change-scores, and can be set differently. A widely used divergence metric, KL-divergence, is defined as

$$
D_{K L}(g \| f)=\int_{x} g(x) \log \left(\frac{g(x)}{f(x)}\right) d x .
$$

It is a non-negative $(\geq 0)$ and non-symmetric measure. It is 0 when the two distributions are completely identical, and becomes larger as the two distributions deviate from each other. The non-symmetry property of the $D_{K L}$ complicates the procedure of setting the threshold for detecting changes in data streams. To overcome the problem of the KL-divergence, this framework provides two 
options on setting divergence metrics. The first divergence metric is a modified symmetric KL-divergence 45

$$
D_{M K L}(\hat{g} \| \hat{f})=\max \left(D_{K L}(\hat{g} \| \hat{f}), D_{K L}(\hat{f} \| \hat{g})\right) .
$$

This divergence metric mimics powerful order selection tests developed in current statistics literature; see, for example, 46].

The second divergence metric is a measure of the intersection area under the curves of two density functions [47,

$$
D_{A}(\hat{g} \| \hat{f})=1-\int_{x} \min (\hat{f}(x), \hat{g}(x)) d x .
$$

This $D_{A}$ takes values in $[0,1]$, where the value one means that the two distributions are completely different and zero means the two distributions are identical. This measure can also be computed using numerical integration techniques on the intersection area.

After computing the change-scores on all the PCs, the different change-score values are aggregated by taking the maximum over all values. This is necessary to maintain a single statistical quantity. The maximum is preferable for aggregating the change-score values as it allows for treating any changes happening at any component equally important. Also, when a change happens in a single PC, the change-score will not be affected by the small change-score values obtained from the other PCs.

Dynamic Threshold Settings: Typical statistical tests for change detection start by considering the null hypothesis, which assumes that the data distribution is stationary. A change-score value is then calculated to determine the probability of rejecting the null hypothesis. The most popular technique to reject the null hypothesis is to specify a threshold and declare a change whenever the changescore becomes greater than the threshold. Most existing methods require a userspecified threshold [48, which has two main issues. First, the fixed threshold cannot be used to detect changes in different magnitudes. Second, the threshold is difficult to set, as it is sensitive to the divergence metric, window size, underlying distribution, and change types. In this framework, the threshold is dynamically adjusted in the detection process (Algorithm 3). More details of the setting can be found in 33 .

Performance Evaluation. The above presented change detection framework is evaluated together with two other change detection methods: $k d q$-tree [28] (using $k d q$-tree data structure to model data distribution) and PCA-SPLL [29] (using PCs with small variances for measuring distribution difference). Table 3 presents the results on datasets, which are generated following the same generation mechanism of [28] and contain the same types of changes: $M(\epsilon)$ means varying the mean value, $D(\epsilon)$ means varying the standard deviation, and $C(\epsilon)$ means varying the correlation. Each dataset contains $5 \times 10^{6}$ data samples with changes that occur every $5 \times 10^{4}$ data samples with a total of 99 change points. At each change point, a set of random numbers in the interval $[-\epsilon,-\epsilon / 2] \cup[\epsilon / 2, \epsilon]$ 
Table 3: Evaluation results of Dasu's method ( $k d q$-tree) with Average Absolute Difference (AAD) and KL-divergence metrics, Kuchneva's method (PCA-SPLL), CD-Area, and CD-MKL with KDE-Track as density estimators. The results are in the form $\mathrm{TP} / \mathrm{L} / \mathrm{FP} / \mathrm{FN}$ with $\mathrm{TP}=$ True Positives, $\mathrm{L}=$ Late detections, FP $=$ the False Positives and $\mathrm{FN}=$ the False Negatives. The best results are in bold.

\begin{tabular}{|l|c|c|c|c|c|}
\hline \multirow{2}{*}{ Dataset } & \multicolumn{2}{|c|}{$k d q$-tree [28] } & PCA-SPLL [29] & CD-Area [33] & CD-MKL [33] \\
\cline { 2 - 3 } & $\mathrm{AAD}$ & $\mathrm{KL}$ & & & \\
\hline $\mathrm{M}(0.01)$ & $30 / 15 / 1 / 54$ & $3 / 7 / 0 / 89$ & $10 / 16 / 5 / 73$ & $\mathbf{3 8 / 2 3 / 0 / 3 8}$ & $34 / 31 / 1 / 34$ \\
$\mathrm{M}(0.02)$ & $77 / 14 / 6 / 8$ & $3 / 7 / 0 / 89$ & $18 / 9 / 3 / 72$ & $\mathbf{8 7 / 1 2 / 0 / 0}$ & $89 / 7 / 0 / 3$ \\
$\mathrm{M}(0.05)$ & $98 / 1 / 4 / 0$ & $12 / 21 / 0 / 66$ & $66 / 11 / 9 / 42$ & $\mathbf{9 9 / 0 / 0 / 0}$ & $95 / 0 / 4 / 4$ \\
\hline $\mathrm{D}(0.01)$ & $42 / 18 / 2 / 39$ & $4 / 2 / 0 / 93$ & $29 / 5 / 4 / 65$ & $45 / 29 / 0 / 25$ & $\mathbf{5 4 / 2 5 / 0 / 2 0}$ \\
$\mathrm{D}(0.02)$ & $98 / 1 / 9 / 0$ & $12 / 7 / 0 / 80$ & $85 / 0 / 2 / 14$ & $\mathbf{9 7 / 0 / 1 / 2}$ & $94 / 1 / 2 / 4$ \\
$\mathrm{D}(0.05)$ & $99 / 0 / 2 / 0$ & $24 / 4 / 2 / 71$ & $89 / 2 / 2 / 8$ & $\mathbf{9 9 / 0 / 0 / 0}$ & $98 / 0 / 2 / 1$ \\
\hline $\mathrm{C}(0.1)$ & $67 / 13 / 2 / 19$ & $8 / 4 / 1 / 87$ & $55 / 4 / 2 / 40$ & $68 / 19 / 0 / 12$ & $\mathbf{7 6 / 1 7 / 2 / 6}$ \\
$\mathrm{C}(0.15)$ & $78 / 9 / 5 / 12$ & $8 / 7 / 1 / 84$ & $63 / 6 / 4 / 30$ & $84 / 7 / 1 / 8$ & $\mathbf{8 5 / 9 / 0 / 5}$ \\
$\mathrm{C}(0.2)$ & $96 / 3 / 10 / 0$ & $21 / 5 / 3 / 73$ & $75 / 3 / 3 / 21$ & $\mathbf{9 7 / 1 / 1 / 1}$ & $93 / 2 / 0 / 4$ \\
\hline
\end{tabular}

are generated and added to the distribution's parameters that will be changed. The parameter $\epsilon$ controls the magnitude of the change, where smaller values for $\epsilon$ make changes harder to detect and vice versa.

The presented framework using the maximum KL-divergence (Eq. (13)) is called CD-MKL, and the one using the Area metric (Eq. 14) is called CDArea. The performance of the different methods is measured according to the number of True Positives (TP), Late detections (L), False Positives (FP) and False Negatives (FN). By true positives, we mean the changes that were reported correctly before receiving $2 w$ from the new distribution where $w$ is the window size. Late detections are the changes reported after processing $2 w$ data samples from the new distribution. False positives are changes reported by the method when there are no changes, and false negatives are the missed changes. The window size $w$ is set to $10^{4}$ for all methods.

Results: The experimental results show that the presented framework outperforms $k d q$-tree and PCA-SPLL in terms of the number of correctly detected changes and less false positives. Also, the performance of the framework when using the Area metric (Eq. (14)) outperforms the performance of the framework when using the MKL (Eq. (13)) metrics in most of the evaluation datasets. PCASPLL [29] shows the worst performance, especially for detecting mean shifts.

The second set of experiments evaluates the detection accuracy in highdimensional datasets. As changes become less observable when the data dimensionality increases, we used only datasets with a reasonable magnitude of change $(M(0.05), D(0.05)$, and $C(0.2))$. For each type of change, we generated three datasets with 10,20, and 30 dimensions. The changes in the data distribution affect only two dimensions (variables) and for more complicated change detection cases, we selected the variables that are affected by the change randomly at each change point. 
Table 4: Evaluation results of $k d q$-tree with Average Absolute Difference (AAD) metric, PCA-SPLL, CD-MKL, and CD-Area for (a) changes in Gaussian high-dimensional data (1st 9 datasets), (b) changes in density shape from a list of non-Gaussian distributions (DistCh), and (c) changes in non-linear dependent data streams (DEMC, DDC and SWRL). The best results are in bold.

\begin{tabular}{|l|c|c|c|c|}
\hline Dataset & $k d q$-tree $(\mathrm{AAD})[28$ & PCA-SPLL $[29$ & CD-MKL 33 & CD-Area [33] \\
\hline $\mathrm{M}(10 \mathrm{D})$ & $96 / 1 / 9 / 2$ & $63 / 10 / 10 / 26$ & $\mathbf{9 9 / 0 / 0 / 0}$ & $\mathbf{9 9 / 0 / 0 / 0}$ \\
$\mathrm{M}(20 \mathrm{D})$ & $75 / 7 / 3 / 17$ & $32 / 11 / 1 / 56$ & $96 / 1 / 2 / 2$ & $\mathbf{9 9 / 0 / 0 / 0}$ \\
$\mathrm{M}(30 \mathrm{D})$ & $59 / 8 / 4 / 32$ & $33 / 13 / 2 / 53$ & $97 / 0 / 1 / 2$ & $\mathbf{9 7 / 1 / 1 / 1}$ \\
\hline $\mathrm{D}(10 \mathrm{D})$ & $93 / 3 / 3 / 3$ & $70 / 1 / 1 / 28$ & $\mathbf{9 9 / 0} \mathbf{0 / 0}$ & $\mathbf{9 9 / 0 / 0 / 0}$ \\
$\mathrm{D}(20 \mathrm{D})$ & $87 / 3 / 7 / 9$ & $59 / 0 / 0 / 40$ & $\mathbf{9 9 / 0 / 0 / 0}$ & $\mathbf{9 9 / 0 / 0 / 0}$ \\
$\mathrm{D}(30 \mathrm{D})$ & $78 / 4 / 4 / 17$ & $56 / 1 / 4 / 42$ & $\mathbf{9 6 / 1 / 1 / 2}$ & $96 / 0 / 0 / 3$ \\
\hline $\mathrm{C}(10 \mathrm{D})$ & $64 / 7 / 10 / 28$ & $67 / 1 / 0 / 31$ & $95 / 0 / 5 / 4$ & $\mathbf{9 8 / 1 / 0 / 0}$ \\
$\mathrm{C}(20 \mathrm{D})$ & $29 / 12 / 12 / 58$ & $49 / 0 / 0 / 50$ & $\mathbf{9 8 / 0 / 2 / 1}$ & $95 / 2 / 2 / 2$ \\
$\mathrm{C}(30 \mathrm{D})$ & $11 / 4 / 5 / 84$ & $48 / 0 / 0 / 51$ & $97 / 0 / 2 / 2$ & $\mathbf{9 9 / 0 / 0 / 0}$ \\
\hline DistCh & $99 / 0 / 7 / 0$ & $72 / 0 / 44 / 27$ & $\mathbf{9 9 / 0 / 0 / 0}$ & $\mathbf{9 9 / 0 / 0 / 0}$ \\
\hline DEMC & $84 / 6 / 2 / 9$ & $24 / 15 / 28 / 60$ & $84 / 6 / 3 / 9$ & $\mathbf{8 8 / 0 / 4 / 1 1}$ \\
DDC & $96 / 2 / 3 / 1$ & $19 / 18 / 29 / 62$ & $94 / 1 / 3 / 4$ & $\mathbf{9 7 / 0 / 0 / 2}$ \\
SWRL & $99 / 0 / 6 / 0$ & $23 / 10 / 29 / 66$ & $95 / 0 / 2 / 4$ & $\mathbf{9 9 / 0 / 2 / 0}$ \\
\hline
\end{tabular}

We report the results for the $k d q$-tree method with AAD-divergence only because KL-divergence shows very low accuracy for $2 \mathrm{D}$ data in Table 3 . The results in Table 4 show that CD-Area and CD-MKL are not affected by data dimensionality. As all space partitioning methods, the $k d q$-tree method, suffers from the curse of dimensionality as data dimensionality increases. The PCASPLL has low accuracy again.

The third set of experiments evaluates the detection accuracy in two special cases: (1) changes in the density shape of data streams, and (2) changes in data streams with non-linear dependencies. Four datasets were generated for evaluation, where each dataset contains 100 batches with batch size of $5 \times 10^{4}$, resulting in a total of 99 changes. DistCh is generated by changing the data distribution in consecutive batches, where a distribution is randomly drawn from a list of preset distributions including: standard Normal, highly skewed Normal, bimodal Normal, trimodal Normal, Gamma and Laplace distributions. In each batch, the mean, variance and correlation are kept constant. The remaining three datasets evaluate the detection accuracy on non-linear dependent data streams. The first dataset includes a Disc with EMpty Circle in the middle (DEMC). Changes in consecutive batches are introduced by randomly altering the radius of the empty circle without affecting the mean, variance, or correlation values. The second dataset includes Disc with Dense Circle in the middle (DDC). Nonlinear changes in the distribution are introduced by altering the radius of the dense circle in the center of the data points. The third dataset is a Swiss roll (SWRL) dataset with changes designed by altering the distance between any two consecutive contours of the Swiss roll. 

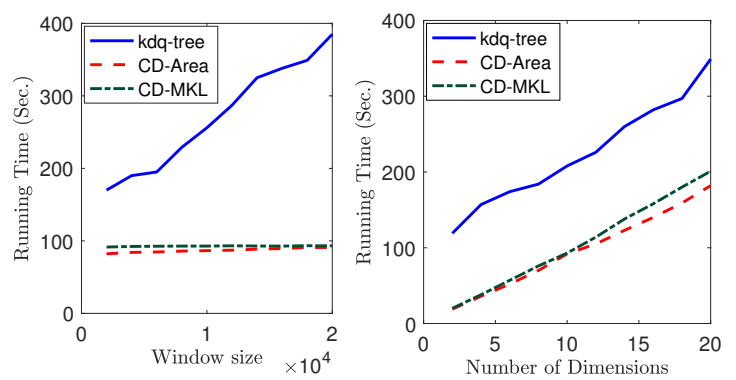

Fig. 9: Running time of the evaluated methods for different window sizes (left) and different number of dimensions (right). The stream size is $\mathbf{5} \times \mathbf{1 0}^{\mathbf{6}}$ data samples. Changes occur every $\mathbf{5} \times \mathbf{1 0}^{\mathbf{4}}$ data samples and the number of changes is 99 .

The results presented in Table 4 show that both CD-MKL and CD-Area outperform other methods in DisCh, and that CD-Area obtained better results in all non-linear dependent data streams. While the experimental results are promising, the proposed method is restricted by PCA's limitation to handling only linearly dependent data streams. Change detection in non-linear dependent data streams will be left for future study.

Computational Cost Analysis: The time complexity of the presented framework and the $k d q$-tree method is linear w.r.t. the size of the data stream. However, the constant in the complexity formula controls the efficiency of the evaluated methods. The cost of the PCA-based framework depends on three main subroutines: 1) The PCA routine for extracting PCs, which is called only when a change is reported. It has a complexity of $O\left(d^{2} \times w \times R_{c}\right)$, where $d$ is the data dimensionality, $w$ is the window size, and $R_{c}$ is the number of reported changes; 2) The incremental density update by KDE-Track, which requires a constant time at the arrival of a new data sample from the stream; and 3) Computing the divergence metric, which is done incrementally and costs a constant time upon the arrival of a new data sample. Therefore, the computational cost of the PCAbased framework is affected more by data dimensionality, as the PCA routine has quadratic complexity w.r.t. dimensionality $d$, and maintaining the density and computing the change-score may have to be done on more PCs when $d$ is higher. The window size has a relatively less effect on the running time of our framework as it affects only the PCA routine.

The running time of the $k d q$-tree method increases fast w.r.t. window size and data dimensionality, mainly due to the expensive bootstrap sampling routine for computing the threshold to report changes. The overall complexity is $O\left(k d w \log \left(\frac{1}{\nu} R_{c}\right)\right)$, where $R_{c}$ is the number of reported changes.

The results in Figure 9 confirm our analysis. We see a slight increase in the running time of CD-Area and CD-MKL when increasing the window size (Figure 9 Left), while the runtime increases almost linearly w.r.t. data dimensionality (Figure 9 Right). The running time of $k d q$-tree increases linearly with the window size and data dimensionality. 
Last, it is worth mentioning that $k d q$-tree requires the data to be normalized (into $[0,1]$ ) before running to ensure that growth of the tree growing is not stopped prematurely by the stopping condition of the minimum cell width. However, it is unrealistic to normalize data streams before hand, as data arrives continuously. The PCA-framework does not require normalization and is thus superior to $k d q$-tree in processing a real data stream.

\section{Summary and Future Work}

Summary. In this chapter, we studied the problem of estimating the dynamic density that comes with data streams. We presented KDE-Track 18 that can timely track the evolving distribution and accurately estimate the probability density function of evolving data streams. KDE-Track models the data distribution as a set of resampling points with their estimated PDF values, which are incrementally updated upon the arrival of new data samples from the stream. The effectiveness and efficiency of KDE-Track have been analytically studied and experimentally demonstrated on both synthetic and real world data.

KDE-Track provides any time available density function, which has been utilized in two main applications. First, the estimated density is used for visualizing and monitoring the changes in New York Taxi pick up data. KDE-Track allows for visualizing the dynamic density estimated from the data in real time after receiving any data sample.

Second, KDE-Track is used to develop a framework for detecting abrupt changes in linearly dependent multidimensional data streams. The framework is based on projecting data on selected principal components. On each projection, densities in reference and test windows are estimated using KDE-Track and compared using effective divergence metrics to compute a change-score value that are used to report change in the data distribution when the score value increase for a reasonable period.

Possible Future Work. First, more applications that benefit from KDE-Track as an accurate and computationally efficient density estimator can be studied. Second, The change detection framework depends mainly on the PCA, which can reflect the changes in linearly dependent data streams. However, more complex changes in nonlinear dependent data streams should be further studied.

\section{References}

1. Zhou, A., Cai, Z., Wei, L., Qian, W.: M-kernel merging: Towards density estimation over data streams. In: DASFAA. (2003)

2. Subramaniam, S., Palpanas, T., Papadopoulos, D., Kalogeraki, V., Gunopulos, D.: Online outlier detection in sensor data using non-parametric models. In: VLDB. (2006)

3. Schaller, B.: A regression model of the number of taxicabs in u. s. cities. Journal of Public Transportation 8 (2005) 63-78 
4. Zhou, Z., Matteson, D.: Predicting ambulance demand: a spatio-temporal kernel approach. In: KDD. (2015)

5. Wu, F., Li, Z., Lee, W., Wang, H., Huang, Z.: Semantic annotation of mobility data using social media. In: WWW. (2015)

6. Scott, D.: Multivariate Density Estimation: Theory, Practice, and Visualization. John Wiley \& Sons (1992)

7. Qahtan, A., Zhang, X., Wang, S.: Efficient estimation of dynamic density functions with an application to outlier detection. In: CIKM. (2012)

8. Babcock, B., Babu, S., Datar, M., Motwani, R., Widom, J.: Models and issues in data stream systems. In: ACM SIGMOD-SIGACT-SIGART. (2002)

9. Zhang, X., Furtlehner, C., Germain-Renaud, C., Sebag, M.: Data stream clustering with affinity propagation. TKDE 26 (2014) 1644-1656

10. Heinz, C., Seeger, B.: Cluster kernels: Resource-aware kernel density estimators over streaming data. TKDE 20 (2008) 880-893

11. Boedihardjo, A.P., Lu, C., Chen, F.: A framework for estimating complex probability density structures in data streams. In: CIKM. (2008)

12. Cao, Y., He, H., Man, H.: SOMKE: Kernel density estimation over data streams by sequences of self-organizing maps. IEEE Transaction on Neural Networks and Learning Systems 23 (2012) 1254-1268

13. Zheng, Y., Jestes, J., Phillips, J., Li, F.: Quality and efficiency in kernel density estimates for large data. In: SIGMOD. (2013)

14. Procopiuc, C., Procopiuc, O.: Density estimation for spatial data streams. In: SSTD. (2005)

15. Gary, A., Moore, A.: Nonparametric density estimation: Toward computational tractability. In: SDM. (2003)

16. LIN, C., WU, J., YEN, C.: A note on kernel polygons. Biometrika 93 (2006) $228-234$

17. Hart, T., Zandbergen, P.: Kernel density estimation and hotspot mapping: Examining the influence of interpolation method, grid cell size, and bandwidth on crime forecasting. Policing: An International Journal of Police Strategies \& Management 37 (2014) 305-323

18. Qahtan, A., Wang, S., Zhang, X.: Kde-track: An efficient dynamic density estimator for data streams. TKDE 29 (March 2017) 642-655

19. Wand, M.: Fast computation of multivariate kernel estimators. Journal of Computational and Graphical Statistics 3 (1994) 433-445

20. Silverman, B.: Density Estimation for Statistics and Data Analysis. Chapman and Hall (1986)

21. Yamanishi, K., Takeuchi, J., Williams, G., Milne, P.: On-line unsupervised outlier detection using finite mixtures with discounting learning algorithms. Data Mining and Knowledge Discovery 8 (2004) 275-300

22. Ke, Y., Sukthankar, R., Hebert, M.: Event detection in crowded videos. In: ICCV. (2007)

23. Kifer, D., Ben-David, S., Gehrke, J.: Detecting change in data streams. In: VLDB. (2004)

24. Aggarwal, C.C.: A framework for diagnosing changes in evolving data streams. In: SIGMOD. (2003)

25. Guralnik, V., Srivastava, J.: Event detection from time series data. In: KDD. (1999)

26. Kawahara, Y., Sugiyama, M.: Change-point detection in time-series data by direct density-ratio estimation. In: SDM. (2009) 
27. Bifet, A., Gavaldà, R.: Learning from time-changing data with adaptive windowing. In: SDM. (2007)

28. Dasu, T., Krishnan, S., Venkatasubramanian, S., Yi, K.: An information-theoretic approach to detecting changes in multi-dimensional data streams. In: Symposium on the Interface of Statistics, Computing Science, and Applications. (2006)

29. Kuncheva, L.I., Faithfull, W.J.: PCA feature extraction for change detection in multidimensional unlabeled data. IEEE Transactions on Neural Networks and Learning Systems 25 (2014) 69-80

30. Song, X., Wu, M., Jermaine, C., Ranka, S.: Statistical change detection for multidimensional data. In: KDD. (2007)

31. Liu, S., Yamada, M., Collier, N., Sugiyama, M.: Change-point detection in timeseries data by relative density-ratio estimation. In: International Conference on Structural, Syntactic, and Statistical Pattern Recognition. (2012) 363-372

32. Takeuchi, J., Yamanishi, K.: A unifying framework for detecting outliers and change points from time series. TKDE 18 (2006) 482-492

33. Qahtan, A.A., Alharbi, B., Wang, S., Zhang, X.: A PCA-Based change detection framework for multidimensional data streams. In: SIGKDD. (2015) 935-944

34. Epanechnikov, V.A.: Non-parametric estimation of a multivariate probability density. Theory of Probability \& Its Applications 14 (1969) 153-158

35. Turlach, B.: Bandwidth selection in kernel density estimation: A review. CORE and Institut de Statistique 19 (1993) 1-33

36. Scott, D., Terrell, G.: Biased and unbiased cross-validation in density estimation. Journal of the American Statistical Association 82 (1987) 1131-1146

37. Hall, P., Sheather, S., Jones, M., Marron, J.: On optimal data-based bandwidth selection in kernel density estimation. Biometrika 78 (1992) 263-269

38. Hall, P., Marron, J.: Estimation of integrated squared density derivatives. Statistics \& Probability Letters 6 (1987) 109-115

39. Jones, M.: The roles of ise and mise in density estimation. Statistics \& Probability Letters 12 (1991) 51-56

40. Shimazaki, H., Shinomoto, S.: Kernel bandwidth optimization in spike rate estimation. J Comput Neurosci. 29 (2010) 171-182

41. Zheng, Y., Phillips, J.: $l_{\infty}$ error and bandwidth selection for kernel density estimates of large data. In: KDD. (2015)

42. Chan, T., Golub, G., LeVeque, R.: Algorithms for computing the sample variance: Analysis and recommendations. The American Statistician 37 (1983) 242-247

43. Sain, R.: Multivariate locally adaptive density estimation. Comput. Stat. Data Anal. 39 (2002) 165-186

44. Marron, J., Wand, M.: Exact mean integrated squared error. The Annals of Statistics 20 (1992) 712-736

45. Liu, D., Sun, D., Qiu, Z.: Feature selection for fusion of speaker verification via maximum kullback-leibler distance. In: ICSP. (2010)

46. Jin, L., Wang, S., Wang, H.: A new nonparametric stationarity test of time series in time domain. Journal of the Royal Statistical Society: Series B 77 (2015) 893-922

47. Cha, S.: Comprehensive survey on distance/similarity measures between probability density functions. Intl. J. of Math. Models and Methods in App. Sci. 1 (2007) 300-307

48. Dai, X.L., Khorram, S.: Remotely sensed change detection based on artificial neural networks. Photogrammetric Engineering \& Remote Sensing 65 (1999) 1179-1186 